\title{
ON THE CRITICAL POINTS OF A MULTIVALENT FUNCTION
}

\author{
BY \\ A. W. GOODMAN
}

1. Introduction. A function $f(z)$, regular in $E$, the open unit circle, cannot be univalent in $E$ if $f^{\prime}(z)=0$ for some $z$ in $E$. The naive generalization of this trivial remark is that a function $f(z)$ regular in $E$ must have valence greater than $p$ if $f^{\prime}(z)$ vanishes for $p$ distinct points $C_{j}$ in $E$. Of course this latter assertion is false, since for each integer $k>0$ it is easy to construct a function which is regular and 2-valent in $E$ and has precisely $k$ critical points (zeros of the derivative) in $E$. Indeed Ahlfors and Grunsky [1] have given an example of a function which is regular and 2-valent in $E$, and has infinitely many critical points there. The proper formulation of the theorem is that $f(z)$ cannot be $p$-valent in $E$ if $k$ of the critical points are too close to the origin. The precise statement is

THEOREM 1. For each positive integer $p$ and for each integer $k \geqq p$, there is a positive number $R^{*}(p, k)$ with the following properties:

A. Let $f(z)$ be regular in $E$ and have critical points $C_{1}, C_{2}, \cdots, C_{k}$ in $E$, where multiple critical points are listed in accordance with their multiplicity. If

$$
\left|C_{j}\right| \leqq R^{*}(p, k), \quad j=1,2, \cdots, k,
$$

and if for at least one index $j$ the strict inequality holds, then $f(z)$ has valence greater than $p$ in $E$.

B. The number $R^{*}(p, k)$ is the largest number with the property $\mathrm{A}$; that is, there exists a function $F(z)$ depending on $p$ and $k$, which is regular and p-valent in $E$, and has $k$ critical points $C_{j}$ for which (1.1) holds with the equality sign for each index $j, j=1,2, \cdots, k$.

For greater clarity, we remark that $f(z)$ and $F(z)$ may have more than $k$ critical points. Our assertion is about the subset of $k$ of these critical points which are closest to the origin.

The proof of the existence of the positive numbers $R^{*}(p, k)$ is an easy consequence of the theory of normal families, and we will give the proof in $\$ 2$. We are more interested in finding the precise value of $R^{*}(p, k)$ and we propose the conjecture that $R^{*}(p, k)$ coincides with

$$
R(p, k)=\left(2 p-1-2\left(p^{2}-p\right)^{1 / 2}\right)^{1 / k}
$$

and that the function

Presented to the Society August 27, 1957; received by the editors February 2, 1957. 


$$
F(z)=F(z, p, k) \equiv \frac{z\left(1-z^{k}\right)^{(2 p-2) / k}}{\left(1+z^{k}\right)^{2 p / k}}
$$

is an extremal function. We will see later that this function is $p$-valent in $E$ and has in $E$ precisely $k$ simple critical points with modulus $R(p, k)$. Of course if $F(z)$ is an extremal function (satisfying condition B of Theorem 1) then so also are $F\left(e^{i \theta} z\right)$ and $a F(z)+b, a \neq 0$. The conjecture is, that aside from these trivial transformations, the extremal function is uniquely given by Equation (1.3).

It is worth noting that if $p=1$, then $R(1, k)=1$ and $F(z, 1, k)=z /\left(1+z^{k}\right)^{2 / k}$ a function which is well-known to be univalent in $E$, and has precisely $k$ critical points on the boundary of $E$. Also one should observe that although we require that $k \geqq p$ in Theorem 1, the function defined by Equation (1.3) is regular in $E$, and $0<R(p, k)<1$. This suggests that there is a related problem in which $k$ may be any positive integer. We discuss this related problem in $\S 2$. In $\S 3$ we construct the example functions $F(z, p, k)$ and obtain properties of these functions. In $\$ 4$ we consider a simple case of this related problem; and for arbitrary $p$, come close to solving this problem; that is we prove that the extremal function must satisfy a certain differential equation (Equation (4.8)) and that the function $G(z)$ Equation (3.1) which we conjecture to be an extremal function for the related problem, does satisfy this differential equation. Only a uniqueness argument for the solutions of Equation (4.8) is missing. In $\$ 5$ we relate these results to a certain coefficient problem for multivalent functions.

2. Existence proofs. A function $f(z)$ is said to be $p$-valent in $E$ if (1) for each complex number $b$, the equation $f(z)=b$ has at most $p$ solutions for $z$ in $E$ and (2) there is some complex $b$ such that this equation has exactly $p$ solutions in $E$. A convergent sequence of functions, each $p$-valent in $E$, need not converge to a $p$-valent function as the example sequence $z+(n+1) z^{2} / 2 n$ shows. Here each element of the sequence is 2 -valent in $E$, and the limit function is 1-valent or univalent in $E$. Since we wish to use the theory of normal families, it is advantageous to drop the second condition in the definition of $p$-valence. Hence throughout this paper a function which is $q$-valent in $E$ will also be considered $p$-valent in $E$ if $q \leqq p$. With this understanding, the family of functions $p$-valent in $E$, is still not normal as the example sequence $1+n z$ shows. We modify the family by requiring that $f(0)=0$ and that $\max \left\{\left|f^{(j)}(0)\right|, j=1,2, \cdots, p\right\}=1$. Let $\mathcal{F}(p)$ denote this modified family of functions. By $[7$, p. 67$]$ the family $\mathcal{F}(p)$ is quasi-normal of order $p$ since each $f(z) \in \mathcal{F}(p)$ assumes the values 0 and 1 each at most $p$ times. Then by $[7$, p. 70] the family $\mathcal{F}(p)$ is normal since for each $f(z) \in \mathcal{F}(p)\left|f^{(j)}(0)\right| \leqq 1$, $j=1,2, \cdots, p$. To prove Theorem 1 it suffices to consider the family $\mathcal{F}(p)$, since for each $f(z)$ regular and $p$-valent in $E$, there are constants $a$ and $b$ such that $a f(z)+b \in \mathcal{F}(p)$ and has the same critical points as $f(z)$. If now, no such constant as described in the theorem exists, then for each integer $n$ there 
would be a function $f_{n}(z) \in \mathcal{F}(p)$ with $k$ of its critical points $C_{j}^{(n)}$ such that $\left|C_{j}^{(n)}\right| \leqq 1 / n, j=1,2, \cdots, k, n=1,2, \cdots$. Since the family $\mathcal{F}(p)$ is normal, there would be a subsequence converging to a limit function $f(z) \in \mathcal{F}(p)$. The limit function could not be the infinite constant since $f_{n}(0)=0$ for each $n$. Further there is some $j, 1 \leqq j \leqq p$ such that $\left|f^{(j)}(0)\right|=1$ since this is the case for each element of $F(p)$. On the other hand $f_{n}(z)$ has at least $k$ critical points in $|z| \leqq 1 / n$ so the limit function must have a critical point of order not less than $k$ at $z=0$. Since $k \geqq p$, this implies $f^{(j)}(0)=0, j=1,2, \cdots, p$. This contradiction establishes Theorem 1 .

It is reasonable to conjecture that if $F(z)$ is any extremal function for Theorem 1, then the map of $E$ under $F(z)$ has $k$-fold symmetry about the origin, and hence $F(z)$ satisfies the equation $e^{2 \pi i / k} F(z)=F\left(z e^{2 \pi i / k}\right)$. If this were so it would follow immediately that $F(z) \neq 0$ in $0<|z|<1$ (hereafter denoted by $\left.E^{\prime}\right)$. For suppose $F\left(z_{0}\right)=0$, with $z_{0} \in E^{\prime}$. Then $F\left(z_{0} e^{2 \pi i j / k}\right)=0$ for $j=1,2, \cdots, k$. These zeros together with $F(0)=0$ give $k+1>p$ zeros for $F(z)$ in $E$ and hence $F(z)$ is not $p$-valent. Similarly if $F(z)$ satisfies the functional equation for $k$-fold symmetry, then the zero at the origin must be a simple zero, and after a suitable rotation we can write

$$
F(z)=z+\sum_{n=1}^{\infty} a_{n} z^{n k+1}
$$

By a well known theorem $[9, \S 2]$ there exists a function $G(z)=z+\cdots \in \mathcal{F}(p)$ such that $F(z)=G\left(z^{k}\right)^{1 / k}$, and $G(z) \neq 0$ in $E^{\prime}$. If $F(z)$ has exactly $k$ critical points in $E$, then $G(z)$ has just one critical point in $E$. Conversely each such $G(z)$ leads to an $F(z) \in \mathcal{F}(p)$ with $k$-fold symmetry where now $k$ may be any positive integer. This suggests that we consider alongside of $\mathcal{F}(p)$ the subset $\mathcal{F}_{0}(p)$ of functions $f(z)=z+\cdots$ which are regular and $p$-valent in $E$, and satisfy the condition $f(z) \neq 0$ for $z$ in $E^{\prime}$. For this family we have

THEOREM 2. For each pair of positive integers $p$ and $k$ there is a positive number $R_{0}^{*}(p, k)$ with the following properiies:

A. Let $g(z)$ be regular in $E$ and have critical points $C_{1}, C_{2}, \ldots, C_{k}$ where multiple critical points are listed in accordance with their multiplicity. If

$$
\left|C_{j}\right| \leqq R_{0}^{*}(p, k), \quad j=1,2, \cdots, k,
$$

and if for at least one index $j$ the strict inequality holds, then $f(z) \oplus F_{0}(p)$.

B. The number $R_{0}^{*}(p, k)$ is the largest number with the property $\mathrm{A}$; that is, there exists a function $F(z) \in \mathcal{F}_{0}(p)$ which has $k$ critical points $C_{j}$ for which (2.1) holds with the equality sign for each index $j, j=1,2, \cdots, k$.

Briefly stated, the condition $f(z) \neq 0$ for $z$ in $E^{\prime}$ permits us to drop the restriction that $k \geqq p$ in Theorem 1 . The same remark about the number of critical points in $E$, made just after Theorem 1, also applies here. We conjecture that $R_{0}^{*}(p, k)=R(p, k)$ given by Equation (1.2) and that except for 
the obvious trivial transformations the extremal function is unique and again given by Equation (1.3).

Of course $\mathcal{F}_{0}(p) \subset \mathcal{F}(p)$ so if the first conjecture is true, the latter follows from it, but not conversely.

To prove Theorem 2 we first remark that the family $\mathcal{F}_{0}(p)$ is quasinormal of order 1 , since each $f(z) \in \mathcal{F}_{0}(p)$ has precisely one zero in $E$, [7, p. 67], and then since $f^{\prime}(0)=1$ the family is normal $[7$, p. 70]. If now no such constant as described in Theorem 2 exists, then a suitably chosen sequence of functions from $\mathcal{F}_{0}(p)$ would converge to an $f(z) \in \mathcal{F}_{0}(p)$ having a multiple zero at the origin, which contradicts the condition $f^{\prime}(0)=1$.

It may be remarked that theorems similar to Theorems 1 and 2 can easily be formulated and proved about the branch points of the image of $E$ under $f(z)$ in the family $\mathcal{F}(p)$ or $\mathcal{F}_{0}(p)$. It seems likely that $F(z, p, k)$ given by Equation (1.3) is also the extremal function for these Theorems. In the interest of brevity however, we do not discuss these bounds on the branch points.

3. The example functions. Since $F(z, p, k)$ given by Equation (1.3) can be written as $G\left(z^{k}\right)^{1 / k}$ where

$$
G(z)=\frac{z(1-z)^{2 p-2}}{(1+z)^{2 p}}=z-(4 p-2) z^{2}+\cdots
$$

it is sufficient to discuss the properties of this function. An easy computation gives

$$
G^{\prime}(z)=\frac{(1-z)^{2 p-3}\left(1-(4 p-2) z+z^{2}\right)}{(1+z)^{2 p+1}}
$$

and from this it is clear that $G^{\prime}(z)$ has only one zero in $E$ and that it is a simple zero at $z=R(p, 1)$. To see that $G(z)$ is $p$-valent in $E$ it is sufficient to remark that $G(z)$ is a rational function of degree $2 p$, and satisfies the functional equation $G(z)=G(1 / z)$. Thus $G(z)$ assumes each value $2 p$ times in the extended complex plane, and therefore it assumes no value more than $p$ times in $E$. It is not necessary to have a precise description of $G(E)$, the image surface of $E$ under $G(z)$, for the work that follows, but the surface may be of interest on its own merits. If $z=e^{i \theta}$ describes the boundary of $E$,

$$
G\left(e^{i \theta}\right)=\frac{(-1)^{p-1}(1-\cos \theta)^{p-1}}{2(1+\cos \theta)^{p}}
$$

describes a doubly covered ray running from the origin to the point at infinity, along the positive real axis when $p$ is odd, and along the negative real axis when $p$ is even. Hence if $b$ is a point not on this ray, $G(E)$ covers this point $p$ times, and if $b$ is on this ray $G(E)$ covers this point $p-1$ times. Since $G(z)$ is real for real $z$, the surface $G(E)$ must be symmetric about the real axis. To visualize this surface, suppose for simplicity that $p=2$ and consider the three pieces; $P_{1}$, a half plane $\Re(w)>0 ; P_{0}$, a full plane slit along the positive real 
axis from $B=1 / 16=G(C)$ to infinity; and $P_{2}$, a half plane $\Re(w)<0$. Join $P_{1}$ and $P_{2}$ along the interval $0<w<1 / 16$, and join $P_{1}$ to $P_{0}$ along the lower bank of the slit $1 / 16<w<\infty$, and $P_{2}$ to $P_{0}$ along the upper bank of this same slit. This gives a surface, with a first order branch point at $B=1 / 16$ and whose boundary consists of the negative real axes for $P_{1}$ and $P_{2}$. Since this surface has all the properties required, and is further symmetrical about the real axis, it is clear that this surface is the surface $G(E)$.

If $p$ is an arbitrary positive integer $P_{1}$ and $P_{2}$ are each to be replaced by a portion of the surface of $\log w$, consisting of $p-1$ half planes. For $P_{1}$, the piece may be visualized as spiralling upward in a counterclockwise manner from the initial half plane $\Re(w)>0$; while for $P_{2}$, the piece may be visualized as spiralling upward in a clockwise manner from the initial half plane $\mathfrak{N}(w)$ $<0$. The base half planes of $P_{1}$ and $P_{2}$ are joined along the interval $0<w<B$ $=G(C)=(1-1 / p)^{p-1} / 4 p$, and $P_{1}$ and $P_{2}$ are each joined as before to $P_{0}$ along the slit $B<w<\infty$. If $p>2$, the pieces $P_{1}$ and $P_{2}$ as real surfaces in 3dimensional space will intersect, but considered as portions of the surface $G(E)$, the pieces $P_{1}$ and $P_{2}$ have in common only the interval $0<w<B$. We can also regard the surface $G(E)$ as being constructed by taking a sector with vertex at the origin and angular aperture $2 \pi(p-1)$, and joining this sector to a base plane $P_{0}$ with a simple branch at $B$, a point on the positive real axis bisecting the sector.

If we wish to proceed from the geometric description of the surface $G(E)$ and deduce from it the form of the mapping function as given by Equation (3.1) we can use a generalized form of the Schwarz-Christoffel transformation developed earlier by the author [4, Equation (2.5)]. In this case it is easier to visualize the situation if we assume that there are $k$ critical points $C_{j}$. We take $k$ congruent sectors each with vertex at the origin. Each of these sectors is attached to a base plane $P_{0}$ with a simple branch point at $B_{j}$ $=G\left(C_{j}\right), j=1,2, \cdots, k$. Since our function is to be $p$-valent it is clear that the angular aperture of each sector cannot be greater than $2 \pi(p-1) / k$, but if these sectors are arranged symmetrically about the origin then we may select $2 \pi(p-1) / k$ as the angular aperture. We assume that the critical points are given by $C_{j}=C \exp (2 \pi i j / k), C>0$; that the vertices of the sectors are images of $\exp (2 \pi i j / k)$; and that the points at infinity for these sectors are images of $\exp (\pi i(2 j+1) / k), j=1,2, \cdots, k$. The exterior angle at the vertex of each sector is $\pi \gamma=\pi(k+2-2 p) / k$. At the point at infinity the lower edge of one sector turns into the upper edge of an adjacent sector, so that the exterior angle for each vertex at infinity is $\pi \gamma=\pi(k+2 p) / k$. Then substituting in Equation (2.5) of [4] we find for the mapping function

$$
\begin{aligned}
S_{k}(z)= & \int_{0}^{z} \prod_{j=1}^{k}\left(1-t e^{2 \pi i j / k}\right)^{(2 p-k-2) / k}\left(1-t e^{\pi i(2 j+1) / k}\right)^{-(k+2 p) / k} \\
& \cdot\left(1-\frac{t}{C_{j}}\right)\left(1-\bar{C}_{j} t\right) d t .
\end{aligned}
$$


The Condition (2.4) of [4] is satisfied, since we have $\sum \gamma_{j}=2(k+1)$, where $k$ is the number of critical points. The integrand for $S_{k}(z)$ can be easily simplified and one finds

$$
S_{k}(z)=\int_{0}^{z}\left(\frac{1-t^{k}}{1+t^{k}}\right)^{2 p / k} \frac{\left(1-t^{k} / C^{k}\right)\left(1-t^{k} C^{k}\right)}{\left(1-t^{2 k}\right)\left(1-t^{k}\right)^{2 / k}} d t .
$$

One might expect that integration of Equation (3.3) would yield $F(z, p, k)$ given by Equation (1.3). A direct computation shows that

$$
\frac{d}{d z} S_{k}(z)=\frac{d}{d z} F(z, p, k)
$$

if and only if

$$
C^{k}+1 / C^{k}=4 p-2,
$$

and since $0<C<1$, this implies $C=R(p, k)$.

It thus appears that the parameter $C>0$ in Equation (3.3) is not arbitrary. If $C \neq R(p, k)$ the sectors will not have their vertices at the origin; if $C<R(p, k)$ the sectors will overlap at $z=0$, and the condition $G(z) \neq 0$ in $0<|z|<1$ will not be satisfied, while if $C>R(p, k)$ the sectors will leave a neighborhood of $z=0$ uncovered.

4. The differential equation for the extremal function. We will apply variational methods to the problem of finding $R_{0}^{*}(p, 1)$ for the family $\mathcal{F}_{0}(p)$. For this we will need the following lemma, giving the change in the zero of a function when the function is given a small variation.

Lemma 1. Let

$$
h^{*}(z)=h(z)+m v(z)+m^{2} r(z, m)
$$

in a neighborhood $N$ of $C$, where $C$ is a simple zero of $h(z), h(z)$ and $v(z)$ are regular in $N$, and $r(z, m)$ is regular for $z$ in $N$, and uniformly bounded for $m$ sufficiently small. Then $h^{*}(z)$ has a simple zero $C^{*}$ in $N$ and

$$
C^{*}=C-\frac{m v(C)}{h^{\prime}(C)}+O\left(m^{2}\right)
$$

as $m \rightarrow 0$.

Proof. If $N^{\prime}$ is a sufficiently small neighborhood of $C$, we can write

$$
h^{*}(z)=\sum_{n=1}^{\infty} h_{n}(z-C)^{n}+m \sum_{n=0}^{\infty} v_{n}(z-C)^{n}+m^{2} r(z, m)=H(z)+\rho(z, m),
$$

where $H(z)=h_{1}(z-C)+m v_{0}$ and

$$
\rho(z, m)=\sum_{n=2}^{\infty} h_{n}(z-C)^{n}+m \sum_{n=1}^{\infty} v_{n}(z-C)^{n}+m^{2} r(z, m) .
$$


Now $h_{1}=h^{\prime}(C) \neq 0$ by hypothesis, so $H(z)$ has a single simple zero at $C^{\prime}$ $=C-m v_{0} / h_{1}$. We will apply Rouche's theorem to Equation (4.3). On the circle $z=C^{\prime}+A\left|m^{2}\right| e^{i \theta}$, where $A>0$ is an appropriate constant we have $H(z)$ $=m^{2} A h_{1} e^{i \theta}$, and

$$
|\rho(z, m)| \leqq\left|\frac{m^{2} h_{2}^{2} v_{0}^{2}}{h_{1}^{2}}\right|+\left|\frac{m^{2} v_{1} v_{0}}{h_{1}}\right|+O\left(m^{3}\right) .
$$

Then if $A$ is taken large enough, we have for each $|m|<m_{0},|H(z)|>|\rho(z, m)|$ on this circle. Hence inside this circle $h(z)$ has exactly one zero $C^{*}$.

To shorten the statement of our theorems let $P R_{0}(p, k)$ denote the problem of finding an extremal function in the family $\mathcal{F}_{0}(p)$, where by an extremal function we understand a function $F(z)$ for which the Condition B of Theorem 2 is satisfied.

THEOREM 3. Let $F(z)$ be an extremal function for $P R_{0}(p, 1)$, for which the critical point $C$ nearest to the origin is a simple one. Then $F(z)$ cannot have two distinct simple critical points in $E$.

We note that this theorem says nothing about multiple critical points. It is conceivable that an extremal $F(z)$ may have multiple critical points, and even that the critical point nearest to the origin may be of order greater than one. To prove Theorem 3, we use a formula for the variation of a branch point developed by the author [6, Equation (1.3)]. Let $F(z)$ have simple critical points at $C$ and $C_{1}$, where without loss of generality we may assume $\left|C_{1}\right| \geqq C>0$. Let $B$ and $B_{1}$ be the associated branch points of $F(E)$. We give $B_{1}$ a small displacement $\lambda$. Then by Equation (1.3) of [6], we find that

$$
\begin{aligned}
F^{*}(z)=F(z)-\frac{z F^{\prime}(z)}{2}\left\{\frac{\lambda}{C_{1}^{2} F^{\prime \prime}\left(C_{1}\right)}\right. & \frac{C_{1}+z}{C_{1}-z} \\
& \left.+\operatorname{con}\left(\frac{\lambda}{C_{1}^{2} F^{\prime \prime}\left(C_{1}\right)}\right) \frac{1+\bar{C}_{1} z}{1-\bar{C}_{1} z}\right\}+O\left(\lambda^{2}\right),
\end{aligned}
$$

(where con( ) denotes the conjugate of the quantity enclosed by the parentheses) and $F^{*}(z) / F^{*^{\prime}}(0) \in \mathcal{F}_{0}(p)$. If we write $\lambda=m e^{i \theta}, m>0$ then Lemma 1 can be applied to $h(z)=F^{\prime}(z)$ and we find a new critical point

$$
C^{*}=C-\frac{m v^{\prime}(C)}{F^{\prime \prime}(C)}+O\left(m^{2}\right),
$$

where

$$
v(z)=-\frac{z F^{\prime}(z)}{2}\left\{\frac{e^{i \theta}}{C_{1}^{2} F^{\prime \prime}\left(C_{1}\right)} \frac{C_{1}+z}{C_{1}-z}+\operatorname{con}\left(\frac{e^{i \theta}}{C_{1}^{2} F^{\prime \prime}\left(C_{1}\right)}\right) \frac{1+\bar{C}_{1} z}{1-\bar{C}_{1} z}\right\} .
$$

Since $F^{\prime}(C)=0$, we find from Equations (4.5) and (4.6) 


$$
C^{*}=C+\frac{m C}{2}\left\{\frac{e^{i \theta}}{C_{1}^{2} F^{\prime \prime}\left(C_{1}\right)} \frac{C_{1}+C}{C_{1}-C}+\operatorname{con}\left(\frac{e^{i \theta}}{C_{1}^{2} F^{\prime \prime}\left(C_{1}\right)}\right) \frac{1+\bar{C}_{1} C}{1-\bar{C}_{1} C}\right\}+O\left(m^{2}\right) .
$$

But $C>0$ and $C$ is minimal, hence $\Re\left(C^{*}-C\right) \leqq 0$ for all $m$ sufficiently small. If, in Equation (4.7), we replace the last term in the braces by its conjugate the real part is not altered. Then since $\theta$ is an arbitrary real number, and $m$ can be taken as small as we wish, the condition $\Re\left(C^{*}-C\right) \leqq 0$ applied to Equation (4.7) yields

$$
\frac{C_{1}+C}{C_{1}-C}+\frac{1+C_{1} C}{1-C_{1} C}=0 .
$$

This implies $C_{1}\left(1-C^{2}\right)=0$, which is impossible.

If we consider only 2-valent functions then all the critical points are simple. This gives immediately

Corollary 1. If $F(z)$ is an extremal function for $P R_{0}(2,1)$ then $F(z)$ has exactly one critical point in $E$.

THEOREM 4. Let $F(z)$ be an extremal function for $P R_{0}(p, 1)$ and assume that for $F(z)$ the closest critical point to the origin is a simple one at $z=C>0$. Then $F(z)$ satisfies the differential equation

$$
\sum_{j=1}^{s} \frac{1}{z_{j}\left(1+(C+1 / C) z_{j}+z_{j}^{2}\right) F^{\prime}\left(z_{j}\right)^{2}}=0,
$$

where the sum is over all the roots $z_{j}$ in $E$ of the equation $F(z)=b$, and the Equation (4.8) holds for each complex $b \neq 0$, which is neither a limit value of $F(z)$ as $|z| \rightarrow 1$, nor a branch point of the surface $F(E)$. Further

$$
\frac{C F^{\prime \prime \prime}(C)}{2 F^{\prime \prime}(C)}+\frac{1-2 C^{2}}{1-C^{2}}=0 .
$$

Proof. We apply to $F(z)$ a variation formula due to Schiffer [10, 5 Equation (3.3)]. This gives

$$
\begin{aligned}
& F^{*}(z)=F(z)+\lambda\left\{\frac{A F^{2}(z)}{F(z)-b}-A F(z) \sum_{j=1}^{s} \frac{L_{1}\left(z_{j}\right)}{z_{j}}\right. \\
& \left.+\bar{A} z F^{\prime}(z) \sum_{j=1}^{s} \frac{z}{1-\bar{z}_{j} z} \operatorname{con}\left(L_{1}\left(z_{j}\right)\right)+A z F^{\prime}(z) \sum_{j=1}^{s} \frac{1}{z_{j}-z} L_{1}\left(z_{j}\right)\right\}+O(\lambda)^{2},
\end{aligned}
$$

and $F^{*}(z) \in \mathcal{F}_{0}(p)$. Here $A$ is an arbitrary complex constant, $b$ satisfies the conditions of the theorem, $L_{1}(z) \equiv F^{2}(z) / z F^{\prime}(z)^{2}$, and $\lambda>0$. We apply Lemma 1 with $h(z)=F^{\prime}(z)$ and $m=\lambda$. A simple computation using $F^{\prime}(C)=0$ gives

$$
\frac{v^{\prime}(C)}{F^{\prime \prime}(C)}=-A \sum_{j=1}^{s} \frac{C}{C-z_{j}} L_{1}\left(z_{j}\right)+\bar{A} \sum_{j=1}^{s} \frac{C^{2}}{1-\bar{z}_{j} C} \operatorname{con}\left(L_{1}\left(z_{j}\right)\right)
$$


If $F(z)$ is an extremal function and $C>0$, then $\Re\left(v^{\prime}(C) / F^{\prime \prime}(C)\right) \geqq 0$. In Equation (4.11) the second sum may be replaced by its conjugate without altering the real part. Then since $A$ is an arbitrary complex constant, this yields

$$
\sum_{j=1}^{s} \frac{C\left(1-C^{2}\right) L_{1}\left(z_{j}\right)}{\left(C-z_{j}\right)\left(1-z_{j} C\right)}=0 .
$$

Using the definition of $L_{1}(z)$ and the fact that $F\left(z_{j}\right)=b$ for each index $j$, it is easy to see that Equation (4.12) is equivalent to Equation (4.8).

To prove that $F(z)$ satisfies Equation (4.9) we vary the branch point $B=G(C)$ using Equation (1.3) of [6], and obtain another neighboring function

$$
\begin{aligned}
F^{*}(z)=F(z)-\frac{z F^{\prime}(z)}{2}\left\{\frac{\lambda}{C^{2} F^{\prime \prime}(C)}\right. & \frac{C+z}{C-z} \\
& \left.+\operatorname{con}\left(\frac{\lambda}{C^{2} F^{\prime \prime}(C)}\right) \frac{1+C z}{1-C z}\right\}+O\left(\lambda^{2}\right),
\end{aligned}
$$

which when divided by $F^{* \prime}(0)$ is also in $\mathcal{F}_{0}(p)$. We apply Lemma 1 with $h(z)=F^{\prime}(z)$ and $\lambda=m e^{i \theta}$. In the computation of $v^{\prime}(C)$ care must be observed with the term $F^{\prime}(z) /(C-z)$, which is regular at $z=C$, since $F^{\prime}(C)=0$. We find that

$$
\begin{aligned}
\frac{v^{\prime}(C)}{F^{\prime \prime}(C)}= & -\frac{C}{2}\left(\frac{e^{i \theta}}{C^{2} F^{\prime \prime}(C)}\right)\left(3+\frac{C F^{\prime \prime \prime}(C)}{F^{\prime \prime}(C)}\right) \\
& +\frac{C}{2} \operatorname{con}\left(\frac{e^{i \theta}}{C^{2} F^{\prime \prime}(C)}\right) \frac{1+C^{2}}{1-C^{2}} .
\end{aligned}
$$

If $F(z)$ is an extremal function and $C>0$, then $\Re\left(v^{\prime}(C) / F^{\prime \prime}(C)\right) \geqq 0$. As is now customary, we replace the last term in Equation (4.14) by its conjugate without altering the real part. Then since $\theta$ is an arbitrary real number we have

$$
3+\frac{C F^{\prime \prime \prime}(C)}{F^{\prime \prime}(C)}-\frac{1+C^{2}}{1-C^{2}}=0
$$

which is equivalent to Equation (4.9).

We remark that other variation formulas are available. If we had used Equations (2.41) and (2.44) of [5], we would have obtained formally the same results, but actually a little weaker, since in that case we would have to assume that $F(z)$ has only one critical point in $E$.

THEOREM 5. The function $G(z)$ given by Equation (3.1) satisfies the two Equations (4.8) and (4.9), when $C=R(p, 1)$, the critical point of $G(z)$.

The computations involved in proving that $G(z)$ satisfies Equation (4.9) 
are dull but direct, and therefore can be omitted. On the other hand the unusual nature of the sum in Equation (4.8) precludes the use of direct methods, since the roots $z_{j}$ of $z(1-z)^{2 p-2} /(1+z)^{2 p}=b$ are not elementary functions of $b$ if $p$ is large. To prove that $G(z)$ satisfies Equation (4.8), we first note that for this function $C+1 / C=4 p-2$ and

$$
\frac{G(z)}{G^{\prime}(z)}=\frac{z\left(1-z^{2}\right)}{1-(4 p-2) z+z^{2}},
$$

so that if we introduce $G^{2}\left(z_{i}\right)=b^{2}$ in the numerator of Equation (4.8) we can eliminate $G^{\prime}\left(z_{i}\right)^{2}$. Equation (4.8) is thus equivalent to

$$
\sum_{j=1}^{s} \frac{z_{j}\left(1-z_{j}^{2}\right)^{2}}{\left(1-(4 p-2) z_{j}+z_{j}^{2}\right)^{3}}=0
$$

where $s=p$ if $b$ is not real. It is now convenient to transform the problem to the half plane $H: \Re \zeta>0$, by means of the substitution $z=(\zeta-1) /(\zeta+1)$. If this substitution is made in Equations (3.1) and (4.5), we find that we are to prove that

$$
\sum \frac{\zeta_{j}^{2}\left(\zeta_{j}^{2}-1\right)}{\left((p-1) \zeta_{j}^{2}-p\right)^{3}}=0
$$

where the sum is over all the roots in $H$ of

$$
G(z)=H(\zeta)=\frac{\zeta^{2}-1}{4 \zeta^{2 p}}=b,
$$

with $b$ not real. Now $H(\zeta)$ is an even function of $\zeta$ so each of the $p$ roots in $H$ can be paired with a root in the complement of the closure of $H$, while on the other hand the sum in Equation (4.16) is an even function of $\zeta$ so that if the sum is extended over all the roots of Equation (4.17) the result is merely doubled. Thus it will be sufficient to prove Equation (4.16) when the sum is over all the roots. To simplify matters replace $b$ by $1 / 4 a$ in Equation (4.17) and write instead

$$
\zeta^{2 p}-a \zeta^{2}+a=0 .
$$

For any root $\zeta_{j}$ of this equation, which is not a multiple root, we find easily that

$$
\frac{d \zeta_{j}}{d a}=\frac{\zeta_{j}\left(\zeta_{j}^{2}-1\right)}{2 a\left((p-1) \zeta_{j}^{2}-p\right)} .
$$

The product of all the roots of Equation (4.18) is $a$, hence

$$
\frac{1}{a^{2 p}} \prod_{j=1}^{2 p} \zeta_{j}^{2 p-2}=\frac{1}{a^{2}}
$$


But using Equation (4.18) which the roots $\zeta_{j}$ must satisfy, we have

$$
\frac{1}{a^{2 p}} \prod_{j=1}^{2 p} \frac{a\left(\zeta_{j}^{2}-1\right)}{\zeta_{j}^{2}}=\prod_{j=1}^{2 p} \frac{\zeta_{j}^{2}-1}{\zeta_{j}}=\frac{1}{a^{2}} .
$$

Logarithmic differentiation of Equation (4.21) with respect to $a$ yields

$$
\sum_{j=1}^{2 p} \frac{1}{\zeta_{j}\left(\zeta_{j}^{2}-1\right)} \frac{d \zeta_{j}}{d a}=-\frac{1}{a} .
$$

If we take into account Equation (4.19) we can write this as

$$
\sum_{j=1}^{2 p} \frac{1}{(p-1) \zeta_{i}^{2}-p}=-1
$$

Finally, if we differentiate this equation once more with respect to $a$, and again use Equation (4.19) we obtain Equation (4.16) and the proof of Theorem 5 is complete.

5. A coefficient problem. Let

$$
f(z)=z+\sum_{n=2}^{\infty} a_{n} z^{n}
$$

belong to the family $\mathcal{F}_{0}(p)$. Since the family is normal there is a sequence of constants $M_{n}$ such that if $f(z) \in \mathcal{F}_{0}(p)$ then $\left|a_{n}\right| \leqq M_{n}$ for $n=2,3, \cdots$. More precise information on the order of the sequence $M_{n}$ was obtained by Biernacki $[2,3$ p. 29] but his results are still qualitative. We propose here the conjecture, that if $A_{n}$ is defined by

$$
J(z)=\frac{z(1+z)^{2 p-2}}{(1-z)^{2 p}}=z+\sum_{n=2}^{\infty} A_{n} z^{n}
$$

then $f(z) \in \mathcal{F}_{0}(p)$ implies $\left|a_{n}\right| \leqq A_{n}$, with equality only if $f(z)=e^{i \theta} J\left(z e^{-i \theta}\right)$. It is obvious that $J(z)=-G(-z)$ so the properties of $J(z)$ are known from $\S 3$.

It may be recalled that in an earlier paper, [4] a similar conjecture was advanced for $p$-valent functions for which the moduli of the zeros were prescribed in advance, and the functions had $p$ zeros in $E$.

Consideration of the extremal function [4, Equation (5.16)] and the function $J(z)$ suggests the following general conjecture which includes both of these as special càses. Let

$$
f(z)=z^{q}+\sum_{n=q+1}^{\infty} a_{n} z^{n}
$$

be regular and $p$-valent in $E$, and suppose that in $E^{\prime}, f(z)$ has exactly $t$ zeros $\beta_{j}, j=1,2, \cdots, t$, where $q+t \leqq p$, and where the moduli of the zeros $\left|\beta_{j}\right|=r_{j}$ are fixed. Set 
(5.4) $F_{M}(z)=\frac{z^{q}(1+z)^{2(p-q-t)}}{(1-z)^{2 p}} \prod_{j=1}^{t}\left(1+\frac{z}{\left|\beta_{j}\right|}\right)\left(1+z\left|\beta_{j}\right|\right)=z^{q}+\sum_{n=q+1}^{\infty} A_{n} z^{n}$,

then the conjecture is that $\left|a_{n}\right| \leqq A_{n}$ and that the extremal function is essentially unique. Since $F_{M}(z)$ satisfies the functional equation $F(z)=F(1 / z)$ it is obviously $p$-valent in $E$. If $q+t=p$, then $F_{M}(z)$ given by Equation (5.4) coincides with that given by Equation (5.16) of [4] and in this case the conjecture has been proved valid for each index $n$ if $f(z)$ is starlike [4, Theorem 2]. When $q+t<p$ the techniques used in [4] seem to fail. When $q=1$ and $t=0$, then $F_{M}(z) \doteq J(z)$ given by Equation (5.2) and $f(z) \in \mathcal{F}_{0}(p)$. For simplicity we will consider only this case.

THEOREM 6. Let $F(z)$ of the form (5.1) be an extremal function for the Nth coefficient in the family $\mathcal{F}_{0}(p)$ for which $a_{N}>0$. If $F(z)$ has a simple critical point at $z=C$ then

$$
(N-1) a_{N}=-\sum_{k=1}^{N-1} k\left(\bar{a}_{k} C^{N-k}+\frac{a_{k}}{C^{N-k}}\right) .
$$

Further $J(z)$ given by Equation (5.2) satisfies Equation (5.5) for every index $N>1$.

To prove this theorem, we vary the branch point $B=F(C)$, just as in the proof of Theorem 4. Then $F^{*}(z)$ is given by Equation (4.13), and when multiplied by $F^{* \prime}(0)^{-1}=1+\Re\left(\lambda / C^{2} F^{\prime \prime}(C)\right)+O\left(\lambda^{2}\right)$ is again in $\mathfrak{F}_{0}(p)$. If $a_{N}^{*}$ denotes the $N$ th coefficient for this new function, then

$$
\begin{aligned}
a_{N}^{*}=a_{N} & +a_{N}(1-N) \Re \frac{\lambda}{C^{2} F^{\prime \prime}(C)} \\
& -\sum_{k=1}^{N-1}\left\{\left(\frac{\lambda}{C^{2} F^{\prime \prime}(C)}\right) \frac{k a_{k}}{C^{N-k}}+\operatorname{con}\left(\frac{\lambda}{C^{2} F^{\prime \prime}(C)}\right) k \bar{a}_{k} \bar{C}^{N-k}\right\}+O\left(\lambda^{2}\right) .
\end{aligned}
$$

Since $a_{N}$ is positive and maximal $\Re\left(a_{N}^{*}-a_{N}\right) \leqq 0$. Replacing the last term in the braces by its conjugate does not alter the real part. But $\lambda$ is an arbitrary complex constant, so that Equation (5.6) and the condition $\Re\left(a_{N}^{*}-a_{N}\right) \leqq 0$ give immediately Equation (5.5).

Next let $D_{N}$ denote the right hand side of Equation (5.5) when the function to be considered is $J(z)$. Since the coefficients of $J(z)$ are all real we can write that

$$
\begin{aligned}
\sum_{n=2}^{\infty} D_{n} z^{n} & =-z J^{\prime}(z) \sum_{n=1}^{\infty}\left(C^{n}+\frac{1}{C^{n}}\right) z^{n} \\
& =-z \frac{\left(1+(4 p-2) z+z^{2}\right)(1+z)^{2 p-3}}{(1-z)^{2 p+1}}\left(\frac{C z}{1-C z}+\frac{z / C}{1-z / C}\right) .
\end{aligned}
$$

But for $J(z), C+1 / C=-(4 p-2)$, so this gives 


$$
\sum_{n=2}^{\infty} D_{n} z^{n}=\frac{z^{2}(4 p-2+2 z)(1+z)^{2 p-3}}{(1-z)^{2 p+1}} .
$$

On the other hand

$$
\sum_{n=2}^{\infty}(n-1) A_{n} z^{n}=z^{2} \frac{d}{d z} \frac{J(z)}{z}=z^{2} \frac{d}{d z} \frac{(1+z)^{2 p-2}}{(1-z)^{2 p}} .
$$

When this differentiation is done, the right side of Equations (5.7) and (5.8) coincide, so that the coefficients in the powers series on the left side must be equal.

If it were known that the extremal function has a simple critical point, and that equality holds in (5.5) for the same function for each index $N$, then the solution $F_{C}(z)$ would be uniquely determined as a function of $C$ for Equation (5.5) gives each $a_{N}$ in terms of those with earlier subscripts. In particular, if $C$ is real all the coefficients are real, and then $J(z)$ is uniquely determined by the condition $p=-(C+1 / C-2) / 4$.

If Equation (5.5) is regarded as an equation in $C$, then for each root $C$ with $|C|<1$, there is another $\operatorname{root} C_{1}=1 / \bar{C}$ with $\left|C_{1}\right|>1$. But Equation (5.5) has at most $2 N-2$ roots, so we have proved that if $F(z)$ is an extremal function for the $N$ th coefficient, it cannot have in $E$ more than $N-1$ simple critical points. This was proved earlier by Schiffer [10, p. 360], for critical points of any order, but his proof is more complicated than ours.

In the simple case $N=2$, Equation (5.5) gives

$$
\left|a_{2}\right| \leqq|C|+\frac{1}{|C|}
$$

so that if the lower bound for $|C|$ conjectured in $\$ 2$ were proved, and if it were known that $F(z)$ has a simple critical point then Equation (5.9) would yield the sharp upper bound $\left|a_{2}\right| \leqq 4 p-2$ with equality for $J(z)$.

THEOREM 7. Let $F(z)$ be an extremal function for the Nth coefficient in the family $\Im_{0}(p)$, for which $a_{N}>0$. Then $F(z)$ satisfies the differential equation

$$
\sum_{j=1}^{8} \frac{F\left(z_{j}\right)^{2}}{z_{j}^{2} F^{\prime}\left(z_{j}\right)^{2}}\left\{(N-1) a_{N}+\sum_{k=1}^{N-1} k\left(\bar{a}_{k} z_{j}^{N-k}+\frac{a_{k}}{z_{j}^{N-k}}\right)\right\}=-b_{N},
$$

where $b_{N}$ is defined by $F(z)^{2} /(F(z)-b)=\sum b_{n} z^{n}$, and where the outer sum is over all the roots $z_{j}$ in $E$ of the equation $F(z)=b$, and Equation (5.10) is valid for each $b \neq 0$ which is not a limit value of $F(z)$ as $|z| \rightarrow 1$, nor a branch point of $F(E)$. Further the function $J(z)$ given by Equation (5.2) satisfies Equation (5.10) for every index $N>1$.

The differential Equation (5.10) for the extremal function was obtained earlier by Schiffer [10, Equation (47)], but he made no conjecture as to the solution of this equation. For completeness we include his proof here. In 
the first draft of this paper, the author proved only that $J(z)$ satisfies Equation (5.10) in the simple case $N=2$, using the method of Theorem 5. However this method seems too laborious for arbitrary $N$, and P. T. Bateman pointed out to me that the Cauchy integral formula leads to a really simple proof that $J(z)$ satisfies Equation (5.10) for arbitrary $N$, and we give below Bateman's proof.

First suppose $F(z)$ is an extremal function for the $N$ th coefficient with $a_{N}>0$. We apply to $F(z)$ the variation formula due to Schiffer [10, 5 Equation (3.3) ]. This gives $F^{*}(z)=z+\sum a_{\mathbf{z}}^{*} z^{k} \in \mathcal{F}_{0}(p)$ where $F^{*}(z)$ is given by Equation (4.10). For the new $N$ th coefficient we have

$$
\begin{aligned}
a_{N}^{*}=a_{N}+\lambda\left\{A b_{n}-A a_{n} \sum_{j=1}^{s} \frac{L_{1}\left(z_{j}\right)}{z_{j}}+\right. & \bar{A} \sum_{j=1}^{s} \sum_{k=1}^{N-1} k a_{k} \bar{z}_{j}^{N-k-1} \operatorname{con}\left(L_{1}\left(z_{j}\right)\right) \\
& \left.+A \sum_{j=1}^{s} \sum_{k=1}^{N} k a_{k} \frac{L_{1}\left(z_{j}\right)}{z_{j}^{N-k+1}}\right\}+O\left(\lambda^{2}\right) .
\end{aligned}
$$

Since $a_{N}>0$ is maximal and $\lambda>0$ is arbitrarily small, the real part of the quantity in braces must be nonpositive. The second sum may be replaced by its conjugate, and then $A$ is a common factor. The fact that $A$ is an arbitrary complex number kads immediately to Equation (5.10).

To prove that $J(z)$ satisfies Equation (5.10) observe that $Q$, the left side of this equation can be written as a contour integral,

$$
\begin{aligned}
Q= & \frac{1}{2 \pi i} \int_{|z|=1-\epsilon} \frac{F^{2}(z)}{z^{2} F^{\prime}(z)^{2}} \\
& \cdot\left\{(N-1) a_{N}+\sum_{k=1}^{N-1} k\left(\bar{a}_{k} z^{N-k}+\frac{a_{k}}{z^{N-k}}\right)\right\} \frac{F^{\prime}(z)}{F(z)-b} d z-r(0)-r(C),
\end{aligned}
$$

where $r(0)$ is the residue of the integrand at $z=0, r(C)$ is the sum of the residues at the critical points of $F(z)$ in $E$, and $\epsilon>0$ is so small that $F(z)-b$ has no zeros in the annulus $1-\epsilon<|z|<1$. For $F(z)=J(z)$, the coefficients are all real, and the only critical point for $J(z)$ in $E$ is a simple one at $z=C=R(p, 1)$. In this case Equation (5.12) can be written

$$
\begin{aligned}
Q= & \frac{1}{2 \pi i} \int_{|z|-1} \frac{1-z^{2}}{z\left(1+(4 p-2) z+z^{2}\right)} \\
& \cdot\left\{(N-1) A_{N}+\sum_{k=1}^{N-1} k\left(A_{k} z^{N-k}+\frac{A_{k}}{z^{N-k}}\right)\right\} \frac{J(z)}{J(z)-b} d z-r(0)-r(C)
\end{aligned}
$$

since in this form it is obvious that the integrand is regular on the boundary, for $p>1$. Moreover $J(z)=J(1 / z)$ so that if $\phi(z)$ denotes the integrand in Equation (5.13) then $\phi(z)=-\phi(1 / z) / z^{2}$ and hence 


$$
\int_{|z|=1} \phi(z) d z=\int_{|z|-1} \phi(1 / z) d(1 / z)=-\int_{|z|=1} \phi(z) d z .
$$

Thus the integral in Equation (5.13) is zero, and we have $Q=-r(0)-r(C)$. With respect to the residue at $C$, it is advantageous to examine the integrand in Equation (5.13). Inspection shows that $C$ is a simple zero of the denominator, but the fact that $J(z)$ satisfies Equation (5.5) (Theorem 6) shows that $C$ is at least a simple zero of the quantity in braces, whence $\phi(z)$ is regular at $z=C$, and $r(C)=0$. All that remains is to show that $r(0)=b_{n}$. For this we put the integrand in the form

$$
\begin{aligned}
\phi(z) & =\frac{1}{z^{2} J^{\prime}(z)}\left\{(N-1) A_{n}+\sum_{k=1}^{N-1} k\left(A_{k} z^{N-k}+\frac{A_{k}}{z^{N-k}}\right)\right\} \frac{J^{2}(z)}{J(z)-b} \\
& =\frac{\sum_{k=1}^{N-1} k A_{k} z^{k}+(N-1) A_{N} z^{N}+\sum_{k=N+1}^{2 N-1}(2 N-k) A_{2 N-k} z^{k}}{z^{N+1} \sum_{k=1}^{\infty} k A_{k} z^{k}} \sum_{k=2}^{\infty} b_{k} z^{k} \\
& =\frac{1}{z^{N+1}}\left(1-A_{N} z^{N-1}+\cdots\right)\left(z^{2}+\cdots+b_{n} z^{n}+\cdots\right) .
\end{aligned}
$$

Clearly the coefficient of $1 / z$ is $b_{n}$.

\section{REFERENCES}

1. L. Ahlfors and H. Grunsky, Über die Blochsche Konstante, Math. Z. vol. 42 (1937) pp. 671-673.

2. M. Biernacki, Sur les fonctions multivalentes d'ordre p, C. R. Acad. Sci. Paris, vol. 203 (1936) pp. 449-451.

3. - Les fonctions multivalentes, Hermann and Cie, Paris, 1938.

4. A. W. Goodman, On the Schwarz-Christoffel transformation and p-valent functions, Trans. Amer. Math. Soc. vol. 63 (1950) pp. 204-223.

5. - Variation formulas for multivalent functions, Trans. Amer. Math. Soc. vol. 89 (1958) pp. 295-309.

6. - Variation of the branch points for an analytic function, Trans. Amer. Math Soc. vol. 89 (1958) pp. 277-284.

7. P. Montel, Leçons sur les familles normales de fonctions analytiques et leurs applications, Paris, Gauthier-Villars, 1927.

8. Z. Nehari, Conformal mapping, New York, McGraw-Hill, 1952.

9. M. S. Robertspn, Multivalent functions of order p, Bull. Amer. Math. Soc. vol. 44 (1938) pp. 282-285.

10. M. Schiffer, Variation of the Green function and theory of the p-valued functions, Amer. J. Math. vol. 65 (1943) pp. 341-360.

The Institute for Advanced Study, Princeton, N. J.

The University of Kentucky, LeXington, KY. 